\title{
Conflicting evidence combination based on uncertainty measure and distance of evidence
}

\author{
Wen Jiang* ${ }^{*}$, Miaoyan Zhuang, Xiyun Qin and Yongchuan Tang
}

*Correspondence:
jiangwen@nwpu.edu.cn;
jiangwenpaper@hotmail.com
School of Electronics
and Information,
Northwestern Polytechnical
University, Xi'an 710072,
Shaanxi, China

${ }^{*}$ Correspondence: jiangwen@nwpuedu.cn; School of Electronics and Information University, Xi'an 710072,

Shaanxi, China

\begin{abstract}
Dempster-Shafer evidence theory is widely used in many fields of information fusion. However, the counter-intuitive results may be obtained when combining with highly conflicting evidence. To deal with such a problem, we put forward a new method based on the distance of evidence and the uncertainty measure. First, based on the distance of evidence, the evidence is divided into two parts, the credible evidence and the incredible evidence. Then, a novel belief entropy is applied to measure the information volume of the evidence. Finally, the weight of each evidence is obtained and used to modify the evidence before using the Dempster's combination rule. Numerical examples show that the proposed method can effectively handle conflicting evidence with better convergence.
\end{abstract}

Keywords: Dempster-Shafer evidence theory, Conflict, Information fusion, Belief entropy, Distance of evidence

\section{Background}

In practical applications, the information collected from the sensors is often imprecise and uncertain. How to deal with the uncertain information effectively is still an open issue. To address the uncertain information, many math tools are presented such as fuzzy sets theory (Zadeh 1965; Jiang et al. 2015), evidence theory (Dempster 1967; Shafer 1977), rough sets theory (Walczak and Massart 1999; Greco et al. 2001), Z numbers (Zadeh 2011; Kang et al. 2012; Jiang et al. 2016e) and D numbers theory (Deng et al. 2014; 2015a). Also sometimes, the methods with mixed intelligent algorithms are used for decision making or related problems (Deng et al. 2015b, c; Jiang et al. 2016b; Deng 2015c).

Dempster-Shafer evidence theory (Dempster 1967; Shafer 1977) (D-S evidence theory) was introduced by Dempster and then developed by Shafer. As an uncertainty reasoning tool, it can efficiently cope with imprecise and uncertain information without prior information, so it is widely used in many fields of information fusion (Jiang et al. 2016c; Deng 2015a). However, the counter-intuitive results may be obtained when dealing with highly conflicting evidence (Zadeh 1986; Jiang et al. 2016d). If this problem cannot be solved effectively, it will greatly limit the application of D-S evidence theory. Many approaches were proposed to resolve the problem (Lefevre et al. 2002; Murphy 
2000; Smets 2007). In general, there are mainly two types of methodologies. One is to modify Dempster's combination rule, and the other is to pre-process the data. Yager (1987) and Smets (2000), solve the problem of counter intuitive combination results through redistribution conflicting evidence. In the work of Murphy (2000), Deng et al. (2004), they pre-processed the data and also achieved ideal effect, based on the Dempster's combination rule.

Among these two methods to solve the counter-intuitive results, according to the first type of methodology, the counter-intuitive behaviors are imputed to the combination rule. However, modifying combination rule usually destroys the good properties such as commutativity and associativity. What's more, modifying the evidence combination rule is blamed to be unreasonable if the counter-intuitive results are caused by sensor failure. Based on the analysis above, researchers prefer to modify data model, in other words, through pre-process evidence to solve the problem of highly conflicting evidence.

As to the method of pre-processing evidence, the weight vector is difficult to determine. At present, most of the researchers are limited to basing on the relationship among evidence or the evidence itself to generate the weight, instead of considering these two aspects. Murphy's method (Murphy 2000) is just a simple arithmetic mean, which does not take into account the relationship and difference among evidence. Deng et al's weighted average method (Deng et al. 2004) uses the distance of evidence to determine the weight, which makes up the shortage of Murphy's method to some degree, but the effect of evidence itself on weight is ignored.

In this paper, a method of modifying the evidence is used to solve the combination problem of conflicting evidence. By considering the difference among evidence and the effect of evidence itself on weight, a combination method based on the distance of evidence (Jousselme et al. 2001) and a novel belief entropy (Deng 2015c) is proposed. First, the novel belief entropy is introduced to measure the uncertainty. Then, according to the distance of evidence, the evidence is divided into credible evidence and incredible evidence, and to be assigned with appropriate weight based on the distance and the uncertainty. Finally, the obtained weight is used to modify the evidence to get the weighted averaging evidence and combine it $(n-1)$ times by the Dempster's combination rule. The experimental results show that the proposed method can effectively deal with the highly conflicting evidence.

This rest of this paper is organized as follows. The basic concepts are briefly introduced in "Preliminaries" section. In "The proposed method" section, a new combination method based on the distance of evidence and Deng entropy is presented. A numerical example is illustrated to show the efficiency of this new method in "Numerical example" section. Finally, a conclusion is presented in "Conclusion" section.

\section{Preliminaries}

In this section, some preliminaries are briefly introduced below.

\section{Dempster-Shafer evidence theory (Dempster 1967; Shafer 1977)}

D-S evidence theory gained increasing interest in the field of information fusion. In this subsection, D-S evidence theory is briefly introduced. 
Definition 1 Let $\Theta$ be the set of $\mathrm{N}$ mutually exclusive and exhaustive hypotheses. This set is called the frame of discernment, and defined as $\Theta=\left\{\theta_{1}, \theta_{2}, \ldots, \theta_{n}\right\}$. The concept of basic probability assignment (BPA) $m: 2^{\Theta} \rightarrow[0,1]$, which is defined as follows:

$$
\left\{\begin{array}{l}
m(\varnothing)=0 \\
\sum_{A \subseteq \Theta} m(A)=1
\end{array}\right.
$$

If $m(A)>0, A$ is called a focal element. The BPA reflects the degree of evidence support for the proposition of $A$ in frame of discernment.

Definition 2 When multiple independent BPAs are available, the combined evidence can be obtained based on the Dempster's combination rule as follows:

$$
m(A)= \begin{cases}\frac{\sum_{B \cap C=A} m_{1}(B) m_{2}(C)}{1-k} & A \neq \varnothing \\ 0 & A=\varnothing\end{cases}
$$

where $k=\sum_{B \cap C=\varnothing} m_{1}(B) m_{2}(C)$, represents the degree of conflict between $m_{1}$ and $m_{2}$. The greater the $k$, the greater the degree of conflict; the smaller the $k$, the smaller the degree of conflict. $k=0$ corresponds to the evidence do no conflict; whereas $k=1$ implies complete contradiction, i.e., none of the combining masses intersect, hence the combination between $m_{1}$ and $m_{2}$ does not exist.

\section{Jousselme distance (Jousselme et al. 2001)}

Jousselme distance is defined with the BPAs as a particular case of vectors in a $2^{|\Theta|}$-linear space. It is an appropriate measure of the difference-or the lack of similarity-between any two BPAs. In this subsection, Jousselme distance is briefly introduced.

Definition 3 Let $m_{1}$ and $m_{2}$ be two BPAs on the same frame of discernment $\Theta$. The distance between $m_{1}$ and $m_{2}$ is:

$$
d\left(m_{1}, m_{2}\right)=\sqrt{\frac{1}{2}\left(\vec{m}_{1}-\vec{m}_{2}\right)^{T} \underline{\underline{D}}\left(\vec{m}_{1}-\vec{m}_{2}\right)}
$$

where $\underline{\underline{D}}$ is an $2^{|\Theta|} \times 2^{|\Theta|}$ matrix whose element are

$$
D(A, B)=\frac{|A \cap B|}{|A \cup B|} \quad A, B \in 2^{\Theta}
$$

\section{Ambiguity measure (Jousselme et al. 2006)}

In evidence theory, the common uncertainty includes: nonspecificity measure (NS) (Didier and Prade 1985), aggregated uncertainty measure (AU) (Harmanec and Klir 1994) and ambiguity measure (AM) (Jousselme et al. 2006; Yang and Han 2016). And $\mathrm{AM}$ is widely used in uncertainty measure, which is defined as follows.

Definition 4 Suppose that $\Theta$ be a frame of discernment, $m$ is a BPA, ambiguity measure $(\mathrm{AM})$ is defined as follows: 


$$
A M(m)=-\sum_{\theta \in \Theta} \operatorname{Bet}_{m}(\theta) \log _{2}\left(\operatorname{Bet} P_{m}(\theta)\right)
$$

where

$$
\operatorname{Bet}_{m}(\theta)=\frac{\sum_{B \in \Theta} m(B)}{|B|}
$$

is the Pignistic probability proposed by Smets and Kennes (1994).

\section{Belief entropy (Deng 2015c)}

Recently, a novel belief entropy, named as Deng entropy, is applied to measure the information volume of the evidence. This entropy is the generalization of Shannon entropy (Shannon 1948). It is an efficient way to measure uncertainty, not only under the situation where the uncertainty is represented by a probability distribution, but also under the situation where the uncertainty is represented by BPAs. When the uncertainty is expressed in the form of a probability distribution, the entropy definitely degenerates to Shannon entropy. The related concepts are given below.

Definition 5 Deng entropy is defined as follows:

$$
E_{d}=-\sum_{i} m\left(B_{i}\right) \log \frac{m\left(B_{i}\right)}{2^{\left|B_{i}\right|}-1}
$$

where $B_{i}$ is a proposition in BPAs $m$, and $\left|B_{i}\right|$ is the cardinality of $B_{i}$.

Specially, this entropy can definitely degenerate to the Shannon entropy if the belief is only assigned to single element. Namely,

$$
E_{d}=-\sum_{i} m\left(C_{i}\right) \log \frac{m\left(C_{i}\right)}{2^{\left|C_{i}\right|}-1}=-\sum_{i} m\left(C_{i}\right) \log m\left(C_{i}\right)
$$

In addition, the belief entropy has been used in Jiang et al. (2016a) and Yuan et al. (2016). For more detailed information, please refer to Deng (2015c) and Fei et al. (2015).

\section{The proposed method}

Suppose that there are $n$ evidence $m_{i} i=1, \ldots, n$, the pre-processing of the evidence can be illustrated as:

$$
\left\{\begin{array}{l}
m=\sum_{i=1}^{n} w_{i} m_{i} \\
\sum_{i=1}^{n} w_{i}=1
\end{array}\right.
$$

where $w_{i}$ is the corresponding weight of evidence $m_{i}$.

In Eq. (9), each $w_{i} m_{i}$ can be considered as the discounted $m_{i}$, and $m$ denotes the weighted averaging evidence of the original $n$ evidence. The $n$ evidence are weighted average according to all the available focal elements, respectively. But how to get the 
appropriate weight? We argue that both the distance of evidence and the uncertainty should be used to generate the appropriate weight of evidence.

The uncertainty (Jousselme et al. 2006) of evidence can reflect the clarity of the evidence: the smaller the uncertainty of evidence, the clearer the evidence, and the higher the credibility. Based on the speciality of uncertainty, we take the uncertainty as one of the factors to determine the credibility of the evidence, then it is used to determine the weight.

In D-S evidence theory, AM is widely used in uncertainty measure. However, to a certain degree, AM may be lack of information in the Pignistic probability conversion process. Compared to the AM, the calculation of the novel belief entropy (Deng 2015c) is more convenient, and can better measure the uncertainty of the evidence.

A example is given to show the effectiveness of this belief entropy below.

Example 1 Assume there are two BPAs in the frame of discernment $\Theta=\{a, b, c\}$, and the BPAs are listed as follows:

$$
\begin{aligned}
& m_{1}: m_{1}(a)=m_{1}(b)=m_{1}(c)=1 / 3 \\
& m_{2}: m_{2}(a)=m_{2}(b)=m_{2}(c)=0.05, \quad m_{2}(a, b, c)=0.85
\end{aligned}
$$

The uncertainty of each method is calculated respectively by Eqs. (5) and (7):

$$
\operatorname{AM}: A M\left(m_{1}\right)=1.5850, A M\left(m_{2}\right)=1.5850 ;
$$

The novel belief entropy: $E_{d}\left(m_{1}\right)=1.5850, E_{d}\left(m_{2}\right)=3.2338$.

From an intuitive point of view, $m_{1}$ has more higher certainty than $m_{2}$, namely, the uncertainty of $m_{2}$ is greater than $m_{1}$. Whereas the AM of these two BPAs is the same, the result of this belief entropy is consistent with intuition.

In order to get the appropriate weight, the novel entropy is used to measure the uncertainty of the evidence, and a new combination method is proposed based on the entropy and the distance of evidence. Meanwhile, some definitions are presented below.

Suppose there are $n$ mutually exclusive evidence.

1. If the Jousselme distance between one evidence and other evidence is small, namely, the evidence is supported by other evidence, then the evidence is considered as credible evidence;

2. If the Jousselme distance between one evidence and other evidence is great, namely, the evidence is not supported by other evidence, then the evidence is considered as incredible evidence.

For a credible evidence, the smaller the entropy, the smaller the uncertainty, then the clearer the evidence and it is favorable to making decision. So, the greater weight should be assigned to the evidence. On the contrary, for a incredible evidence, the smaller the entropy, the smaller the uncertainty, then the clearer the evidence. However, since there is a great conflict between the incredible evidence and other evidence, and in order to weaken its negative effects, the smaller weight should be assigned to the evidence. Based on this idea, a reward function and a penalty function are defined to generate weight. 
Definition 6 A reward function is defined as:

$$
\alpha_{i}=\exp \left(-\overline{E_{d}}\left(m_{i}\right)\right), \quad i=1,2, \ldots, n
$$

where $\overline{E_{d}}\left(m_{i}\right)$ is the normalized belief entropy, namely, $0 \leq \overline{E_{d}}\left(m_{i}\right) \leq 1$.

This presented reward function meets the following properties:

Property 1 The reward function is always positive, i.e., $\alpha_{i}>0$.

Proof According to the mathematic properties of the exp-function, we can get that $\alpha_{i}>0$.

Property 2 The reward function is a monotone decreasing function.

Proof According to the mathematic properties of the exp-function, we can get that this reward function is a monotone decreasing function. Thus, it can achieve that the smaller the entropy, the greater the weight. In the method, the reward function is used to generate the weight for the credible evidence.

Definition 7 A penalty function is defined as:

$$
\alpha_{i}=\exp \left[-\left(\overline{E_{d}^{\max }}+1-\overline{E_{d}}\left(m_{i}\right)\right)\right], \quad i=1,2, \ldots, n
$$

where $\overline{E_{d}^{\max }}$ is the maximum normalized belief entropy. $\overline{E_{d}}\left(m_{i}\right)$ is the normalized belief entropy, namely, $0 \leq \overline{E_{d}}\left(m_{i}\right) \leq 1$.

This presented penalty function meets the following properties:

Property 3 The penalty function is always positive, i.e., $\alpha_{i}>0$.

Proof According to the mathematic properties of the exp-function, we can get that $\alpha_{i}>0$.

Property 4 The penalty function is a monotone increasing function.

Proof Arbitrary take variables $\overline{E_{d}}\left(m_{1}\right)$ and $\overline{E_{d}}\left(m_{2}\right)$, and suppose $\overline{E_{d}}\left(m_{2}\right)>\overline{E_{d}}\left(m_{1}\right)$, that is $\overline{E_{d}}\left(m_{2}\right)-\overline{E_{d}}\left(m_{1}\right)>0$, then

$$
\begin{aligned}
\alpha_{2}-\alpha_{1} & =\exp \left[-\left(\overline{E_{d}^{\max }}+1-\overline{E_{d}}\left(m_{2}\right)\right)\right]-\exp \left[-\left(\overline{E_{d}^{\max }}+1-\overline{E_{d}}\left(m_{1}\right)\right)\right] \\
& =\exp \left[\left(\overline{E_{d}}\left(m_{2}\right)-\overline{E_{d}}\left(m_{1}\right)\right)\right]
\end{aligned}
$$

Since

$$
\overline{E_{d}}\left(m_{2}\right)-\overline{E_{d}}\left(m_{1}\right)>0 .
$$

Based on the mathematic properties of the exp-function, we can get that: 


$$
\exp \left[\left(\overline{E_{d}}\left(m_{2}\right)-\overline{E_{d}}\left(m_{1}\right)\right)\right]>\exp (0)=1 .
$$

Hence

$$
\alpha_{2}-\alpha_{1}>0, \quad \text { i.e., } \alpha_{2}>\alpha_{1} .
$$

Namely, the penalty function is a monotone increasing function. So it can achieve that the smaller the entropy, the smaller the weight. In the method, the penalty function is used to generate the weight for the incredible evidence.

Assume there are $n$ evidence $m_{i} i=1,2, \ldots, n$, the building steps of weight are given below:

Step 1 Obtain the Jousselme distances $d_{i j} i, j=1,2, \ldots, n$ of every two evidence $m_{i}$ and $m_{j}$ by Eq. (3). The distance matrix $D M$ is given as follows:

$$
D M=\left[d_{i j}\right]=\left[\begin{array}{cccc}
0 & d_{12} & \cdots & d_{1 n} \\
d_{21} & 0 & \cdots & d_{2 n} \\
\vdots & \vdots & \vdots & \vdots \\
d_{n 1} & d_{n 2} & \cdots & 0
\end{array}\right]
$$

Step 2 Calculate the average evidence distance $\overline{d_{i}}$ of the evidence $m_{i}$,

$$
\bar{d}_{i}=\frac{\sum_{j=1, j \neq i}^{n} d_{i j}}{n-1}, \quad i, j=1,2, \ldots, n
$$

Step 3 Calculate the global evidence distance $d$.

$$
d=\frac{\sum_{i=1}^{n} \overline{d_{i}}}{n}, \quad i=1,2, \ldots, n
$$

Step 4 The evidence set is divided into two parts: the credible evidence and the incredible evidence.

If $\overline{d_{i}} \leq d$, then $m_{i}$ is a credible evidence;

If $\overline{d_{i}}>d$, then $m_{i}$ is a incredible evidence.

Step 5 Calculate the belief entropy $E_{d}\left(m_{i}\right), i=1,2, \ldots, n$, and normalize it as follows.

$$
\overline{E_{d}}\left(m_{i}\right)=\frac{E_{d}\left(m_{i}\right)}{\sum_{i=1}^{n} E_{d}\left(m_{i}\right)}, \quad i=1,2, \ldots, n
$$

Step 6 Obtain the corresponding initial weight $\alpha_{i}, i=1,2, \ldots, n$ as follows:

1. For the credible evidence, the reward function is used to generate the initial weight $\alpha_{i}$ according to Eq. (10);

2. For the incredible evidence, the penalty function is used to generate the initial weight $\alpha_{i}$ according to Eq. (11).

Step 7 The final weight $w_{i}$ of evidence $m_{i}$ are normalized as follows:

$$
w_{i}=\frac{\alpha_{i}}{\sum_{i=1}^{n} \alpha_{i}}, \quad i=1,2 \ldots, n
$$


According to Eq. (9), we can get the weighted averaging evidence by the weighted average method of multi-source evidence after obtaining the weight of each evidence. Finally, the new evidence is combined for $(n-1)$ times by Dempster's combination rule and the fusion result can be obtained.

It should be pointed out that, if there are only two BPAs, the Jousselme distance loses efficacy, the building steps of weight are given as follows:

Step 1 Calculate the belief entropy $E_{d}\left(m_{i}\right), i=1,2$, and normalize it by Eq. (15).

Step 2 The weight $\alpha_{i 1}$ is obtained according to the reward function.

$$
\alpha_{i 1}=\exp \left(-\overline{E_{d}}\left(m_{i}\right)\right), \quad i=1,2
$$

Step 3 The weight $\alpha_{i 2}$ is obtained according to the penalty function.

$$
\alpha_{i 2}=\exp \left[-\left(\overline{E_{d}^{\max }}+1-\overline{E_{d}}\left(m_{i}\right)\right)\right], \quad i=1,2
$$

where $\overline{E_{d}^{\max }}$ is the maximum normalized belief entropy.

Step 4 A average weight $\alpha_{i}$ is given as follows:

$$
\alpha_{i}=\frac{1}{2}\left(\alpha_{i 1}+\alpha_{i 2}\right), \quad i=1,2
$$

Step 5 The final weight $w_{i}, i=1,2$ of evidence $m_{i}, i=1,2$ are normalized by Eq. (16).

\section{Numerical example}

In this section, a simple example is given to show the efficiency of the new method.

Example 2 In a multisensor-based target recognition system, there are totally three types of targets: $\Theta=\{A, B, C\}$. Suppose there are five sensors, and five acquired BPAs are listed as follows:

$$
\begin{aligned}
& m 1: m_{1}(A)=0.41, m_{1}(B)=0.29, m_{1}(C)=0.30 ; \\
& m 2: m_{2}(A)=0.00, m_{2}(B)=0.90, m_{2}(C)=0.10 ; \\
& m 3: m_{3}(A)=0.58, m_{3}(B)=0.07, m_{3}(A, C)=0.35 ; \\
& m 4: m_{4}(A)=0.55, m_{4}(B)=0.10, m_{4}(A, C)=0.35 ; \\
& m 5: m_{5}(A)=0.60, m_{5}(B)=0.10, m_{5}(A, C)=0.30 .
\end{aligned}
$$

Firstly, according to Eq. (3), the Jousselme distance of every two evidence can be calculated based on the initial evidence, and the distance matrix $D M$ can be obtained as follows:

$$
D M=\left[\begin{array}{ccccc}
0 & 0.5386 & 0.2892 & 0.2699 & 0.2848 \\
0.5386 & 0 & 0.7195 & 0.6901 & 0.7106 \\
0.2892 & 0.7195 & 0 & 0.0300 & 0.0255 \\
0.2699 & 0.6901 & 0.0300 & 0 & 0.0354 \\
0.2848 & 0.7106 & 0.0255 & 0.0354 & 0
\end{array}\right]
$$

Then, adopt Eq. (13) to calculate the average evidence distance $\overline{d_{i}}, i=1,2,3,4,5$, which is given below:

$$
\bar{d}_{1}=0.3456, \quad \bar{d}_{2}=0.6647, \quad \bar{d}_{3}=0.2661, \quad \bar{d}_{4}=0.2564, \quad \bar{d}_{5}=0.2641 .
$$


Secondly, the global evidence distance $d$ can be obtained by Eq. (14):

$$
d=0.3594
$$

Thirdly, from the above, we can know that $m_{1}, m_{3}, m_{4}, m_{5}$ are the credible evidence and $m_{2}$ is the incredible evidence.

Additionally, the belief entropy $E_{d}\left(m_{i}\right), i=1,2,3,4,5$ is calculated according to Eq. (7):

$$
\begin{array}{ll}
E_{d}\left(m_{1}\right)=1.5664, & E_{d}\left(m_{2}\right)=0.4690, \\
E_{d}\left(m_{4}\right)=1.8914, & E_{d}\left(m_{5}\right)=1.7710 .
\end{array}
$$

The results after normalization of this entropy $\overline{E_{d}}\left(m_{i}\right), i=1,2,3,4,5$ according to Eq. (15) are as follows:

$$
\begin{array}{ll}
\overline{E_{d}}\left(m_{1}\right)=0.2087, \quad \overline{E_{d}}\left(m_{2}\right)=0.0625, \quad \overline{E_{d}}\left(m_{3}\right)=0.2410, \\
\overline{E_{d}}\left(m_{4}\right)=0.2520, \quad \overline{E_{d}}\left(m_{5}\right)=0.2359 .
\end{array}
$$

Then, the weight $\alpha_{i}, i=1,2,3,4,5$ of the credible evidence and the incredible evidence can be obtained based on Eqs. (10) and (11), respectively.

$$
\begin{aligned}
& \alpha_{1}=\exp (-0.2087)=0.8117 \\
& \alpha_{2}=\exp [-(0.2520+1-0.0625)]=0.3044 \\
& \alpha_{3}=\exp (-0.2410)=0.7858 \\
& \alpha_{4}=\exp (-0.2520)=0.7773 \\
& \alpha_{5}=\exp (-0.2359)=0.7899
\end{aligned}
$$

According to Eq. (16), the final weight $w_{i}, i=1,2,3,4,5$ after normalization is shown as follows:

$$
\begin{array}{ll}
w_{1}=0.2340, & w_{2}=0.0877, \quad w_{3}=0.2265, \\
w_{4}=0.2241, & w_{5}=0.2277 .
\end{array}
$$

Obviously, we can obtain that there is a highly conflict between the evidence $m_{2}$ and other evidence. So $m_{2}$ is defined as an incredible evidence, and its weight is only 0.0877 . Other evidence is supported by each other, so their weight are higher than $m_{2}$.

Finally, use the weight to modify the evidence, the results are:

$$
m(A)=0.4872, \quad m(B)=0.2078, \quad m(C)=0.0790, \quad m(A, C)=0.2260 .
$$

After combine for 4 times by Dempster's combination rule, the final results are given below:

$$
m(A)=0.9837, \quad m(B)=0.0021, \quad m(C)=0.0110, \quad m(A, C)=0.0032 .
$$

The fusing results derived based on different combination rules are listed in Table 1. As illustrated in Table 1, when conflicting evidence is acquired, Dempster's combination rule produces counter-intuitive results. When more BPAs are available, Murphy's method (Murphy 2000), Deng et al's method (Deng et al. 2004) and the proposed method in this paper all provide reasonable results. However, Murphy's method is only a simple arithmetic mean, without taking the relationship among the evidence into account. Deng 
Table 1 Evidence combination results based on different combination methods

\begin{tabular}{lllllll}
\hline Evidence & Method & $\boldsymbol{m}(\boldsymbol{A})$ & $\boldsymbol{m}(\boldsymbol{B})$ & $\boldsymbol{m}(\boldsymbol{C})$ & $\boldsymbol{m}(\boldsymbol{A C})$ & Target \\
\hline$m_{1}, m_{2}$ & Dempster's method (Dempster 1967) & 0 & 0.8969 & 0.1031 & 0 & $\mathrm{~B}$ \\
& Murphy's method (Murphy 2000) & 0.0964 & 0.8119 & 0.0917 & 0 & $\mathrm{~B}$ \\
& Deng et al.'s method (Deng et al. 2004) & 0.0964 & 0.8119 & 0.0917 & 0 & $\mathrm{~B}$ \\
& The proposed method & 0.0964 & 0.8119 & 0.0917 & 0 & $\mathrm{~B}$ \\
$m_{1}, m_{2}, m_{3}$ & Dempster's method (Dempster 1967) & 0 & 0.6575 & 0.3425 & 0 & $\mathrm{~B}$ \\
& Murphy's method (Murphy 2000) & 0.4939 & 0.4180 & 0.0792 & 0.0090 & $\mathrm{~A}$ \\
& Deng et al.'s method (Deng et al. 2004) & 0.4974 & 0.4054 & 0.0888 & 0.0084 & $\mathrm{~A}$ \\
& The proposed method & 0.7614 & 0.1295 & 0.0961 & 0.0130 & $\mathrm{~A}$ \\
$m_{1}, m_{2}, m_{3}, m_{4}$ & Dempster's method (Dempster 1967) & 0 & 0.3321 & 0.6679 & 0 & $\mathrm{C}$ \\
& Murphy's method (Murphy 2000) & 0.8362 & 0.1147 & 0.0410 & 0.0081 & $\mathrm{~A}$ \\
& Deng et al.'s method (Deng et al. 2004) & 0.9089 & 0.0444 & 0.0379 & 0.0089 & $\mathrm{~A}$ \\
& The proposed method & 0.9379 & 0.0173 & 0.0361 & 0.0087 & $\mathrm{~A}$ \\
$m_{1}, m_{2}, m_{3}, m_{4}, m_{5}$ & Dempster's method (Dempster 1967) & 0 & 0.1422 & 0.8578 & 0 & $\mathrm{C}$ \\
& Murphy's method (Murphy 2000) & 0.9620 & 0.0210 & 0.0138 & 0.0032 & $\mathrm{~A}$ \\
& Deng et al.'s method (Deng et al. 2004) & 0.9820 & 0.0039 & 0.0107 & 0.0034 & $\mathrm{~A}$ \\
& The proposed method & 0.9837 & 0.0021 & 0.0110 & 0.0032 & $\mathrm{~A}$ \\
\hline
\end{tabular}

et al's method uses the distance of evidence to determine the weight, which makes up the shortage of Murphy's method, but the effect of evidence itself on weight is ignored. The experimental results show that the proposed method performs better than other methods. The reason is that the proposed method can better measure the uncertainty of evidence by Deng entropy and takes into consider the relationship between evidence and the evidence itself to define weight. Furthermore, it can strengthen the effect of credible evidence further and at the same time weaken the effect of incredible evidence further by the reward function and the penalty function.

\section{Conclusion}

Dempster's combination rule will generate counter-intuitive results when dealing with highly conflicting evidence. In the past, more attention was paid to the relationship among the evidence in information fusion, and the research on the evidence itself is ignored. In the presented method, a novel belief entropy is taken as the uncertainty measure, and it is more accurate than the AM. Furthermore, the new method can efficiently handle conflicting evidence with better performance of convergence by jointly using the distance of evidence and the uncertainty measure.

In the future work, more factors will be analyzed and used in establishing the weight to construct more powerful evidence combination methods.

Authors' contributions

WJ designed all the research. WJ and MZ analyzed the data and wrote the manuscript. $M Z, X Q$ and $Y T$ performed the experiments. All authors read and approved the final manuscript.

Acknowledgements

The work is partially supported by National Natural Science Foundation of China (Grant No. 60904099), Natural Science Basic Research Plan in Shaanxi Province of China (Program No. 2016JM6018), the Seed Foundation of Innovation and Creation for Graduate Students in Northwestern Polytechnical University (Program No. Z2016122). 
Received: 4 March 2016 Accepted: 19 July 2016

Published online: 29 July 2016

\section{References}

Dempster AP (1967) Upper and lower probabilities induced by a multi-valued mapping. Ann Math Stat 38(2):325-339

Deng Y (2015a) Generalized evidence theory. Appl Intell 43(3):530-543

Deng Y (2015b) A threat assessment model under uncertain environment. Math Probl Eng 2015:878024. doi:10.1155/2015/878024

Deng Y (2015c) Deng entropy: a generalized Shannon entropy to measure uncertainty. http://vixra.org/abs/1502.0222

Deng Y, Shi W, Zhu Z, Liu Q (2004) Combining belief functions based on distance of evidence. Decis Support Syst 38(3):489-493

Deng X, Hu Y, Deng Y, Mahadevan S (2014) Supplier selection using AHP methodology extended by D numbers. Expert Syst Appl 41(1):156-167

Deng X, Lu X, Chan FTS, Sadiq R, Mahadevan S, Deng Y (2015a) D-CFPR: D numbers extended consistent fuzzy preference relations. Knowl-Based Syst 73:61-68

Deng Y, Mahadevan S, Zhou D (2015b) Vulnerability assessment of physical protection systems: a bio-inspired approach. Int J Unconv Comput 11(3-4):227-243

Deng Y, Liu Y, Zhou D (2015c) An improved genetic algorithm with initial population strategy for symmetric TSP. Math Probl Eng 2015:212794. doi:10.1155/2015/212794

Didier D, Prade H (1985) A note on measures of specificity for fuzzy sets. Int J Gen Syst 10(4):279-283

Fei L, Deng Y, Mahadevan S (2015) Which is the best belief entropy? http://vixra.org/abs/1511.0145

Greco S, Matarazzo B, Slowinski R (2001) Rough sets theory for multicriteria decision analysis. Eur J Oper Res 129(1):1-47

Harmanec D, Klir GJ (1994) Measuring total uncertainty in Dempster-Shafer theory: a novel approach. Int J Gen Syst 22(4):405-419

Jiang W, Luo Y, Qin X, Zhan J (2015) An improved method to rank generalized fuzzy numbers with different left heights and right heights. J Intell Fuzzy Syst 28(5):2343-2355

Jiang W, Wei B, Xie C, Zhou D (2016a) An evidential sensor fusion method in fault diagnosis. Adv Mech Eng 8(3):1-7. doi:10.1177/1687814016641820

Jiang W, Xie C, Wei B, Zhou D (2016b) A modified method for risk evaluation in failure modes and effects analysis of aircraft turbine rotor blades. Adv Mech Eng 8(4):1-16. doi:10.1177/1687814016644579

Jiang W, Zhan J, Zhou D, Li X (2016c) A method to determine generalized basic probability assignment in the open world. Math Probl Eng 2016:3878634. doi:10.1155/2016/3878634

Jiang W, Wei B, Qin X, Zhan J, Tang Y (2016d) Sensor data fusion based on a new conflict measure. Math Probl Eng 2016:5769061. doi:10.1155/2016/5769061

Jiag W, Xie C, Luo Y, Tang Y (2016e) Ranking z-numbers with an improved ranking method for generalized fuzzy numbers. J Intell Fuzzy Syst. doi:10.3233/JIFS-16139

Jousselme AL, Grenier D, Bosse E (2001) A new distance between two bodies of evidence. Inf Fusion 2(2):91-101

Jousselme A, Liu C, Grenier D, Bosse E (2006) Measuring ambiguity in the evidence theory. IEEE Trans Syst Man Cybern Part A Syst Hum 36(5):890-903

Kang B, Wei D, Li Y, Deng Y (2012) A method of converting Z-number to classical fuzzy number. J Inf Comput Sci 9(3):703-709

Lefevre E, Colot O, Vannoorenberghe P (2002) Belief function combination and conflict management. Inf Fusion 3:149-162

Murphy CK (2000) Combining belief functions when evidence conflicts. Decis Support Syst 29(1):1-9

Shafer G (1976) A mathematical theory of evidence. Princeton University Press, Princeton, NJ

Shannon C (1948) A mathematical theory of communication. Bell Syst Tech J 27(3):379-423

Smets P (2000) Data fusion in the transferable belief model. Inf Fusion 1:21-33

Smets P (2007) Analyzing the combination of conflicting belief functions. Inf Fusion 8(4):387-412

Smets P, Kennes R (1994) The transferable belief model. Artif Intell 66(2):191-234

Walczak B, Massart DL (1999) Rough sets theory. Chemom Intell Lab Syst 47(1):1-16

Yager RR (1987) On the Dempster-Shafer framework and new combination rules. Inf Sci 41(2):93-137

Yang Y, Han D (2016) A new distance-based total uncertainty measure in the theory of belief functions. Knowl-Based Syst 94:114-123

Yuan K, Xiao F, Fei L, Kang B, Deng Y (2016) Modeling sensor reliability in fault diagnosis based on evidence theory. Sensors 16(1):113. doi:10.3390/s16010113.

Zadeh LA (1965) Fuzzy sets. Inf Control 8(3):338-353

Zadeh LA (1986) A simple view of the Dempster-Shafer theory of evidence and its implication for the rule of combination. Al Mag 7(2):85-90

Zadeh LA (2011) A note on z-numbers. Inf Sci 181(14):2923-2932 